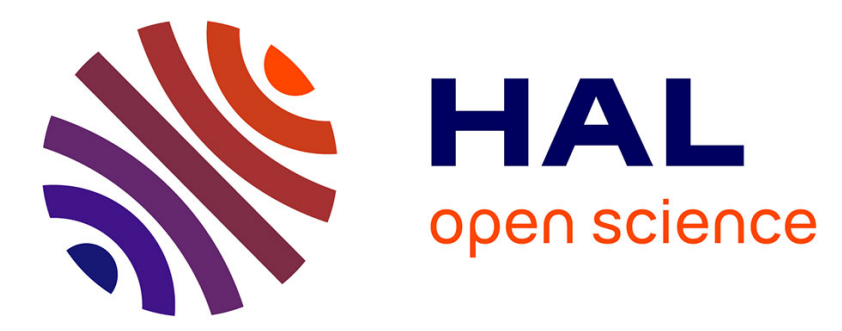

\title{
On the Navier-Stokes equations simulation of the head-on collision between two surface solitary waves
}

Pierre Lubin, Stéphane Vincent, Jean Paul Caltagirone

\section{To cite this version:}

Pierre Lubin, Stéphane Vincent, Jean Paul Caltagirone. On the Navier-Stokes equations simulation of the head-on collision between two surface solitary waves. Comptes Rendus Mécanique, 2005, 333 (4), pp.351-357. 10.1016/j.crme.2005.02.005 . hal-00294106

\section{HAL Id: hal-00294106 https://hal.science/hal-00294106}

Submitted on 8 Jul 2008

HAL is a multi-disciplinary open access archive for the deposit and dissemination of scientific research documents, whether they are published or not. The documents may come from teaching and research institutions in France or abroad, or from public or private research centers.
L'archive ouverte pluridisciplinaire HAL, est destinée au dépôt et à la diffusion de documents scientifiques de niveau recherche, publiés ou non, émanant des établissements d'enseignement et de recherche français ou étrangers, des laboratoires publics ou privés. 


\title{
On the Navier-Stokes equations simulation of the head-on collision between two surface solitary waves
}

\author{
Pierre Lubin a $^{\text {, Stéphane Vincent }}{ }^{\text {b }}$, Jean-Paul Caltagirone ${ }^{\text {c }}$ \\ Transferts, Ecoulements, Fluides, Energétique (TREFLE), UMR CNRS 8508 \\ Site Ecole Nationale Supérieure de Chimie et de Physique de Bordeaux (ENSCPB), Université Bordeaux 1 \\ 16 avenue Pey-Berland, 33607 Pessac Cedex, France. \\ a E-mail: lubin@enscpb.fr (Tél. $0540003307 \quad$ Fax 05400066 68) \\ b E-mail: vincent@enscpb.fr \\ c E-mail: calta@enscpb.fr
}

(Reçu le jour mois année, accepté après révision le jour mois année)

Abstract. The scope of this Note is to show the results obtained for simulating the two-dimensional head-on collision of two solitary waves by solving the Navier-Stokes equations in air and water. The work is dedicated to the numerical investigation of the hydrodynamics associated to this highly nonlinear flow configuration, the first numerical results being analyzed. The original numerical model is proved to be efficient and accurate in predicting the main features described in experiments found in the literature. This Note also outlines the interest of this configuration to be considered as a test-case for numerical models dedicated to computational fluid mechanics. (C) 2005 Académie des sciences/Éditions scientifiques et médicales Elsevier SAS

Computational fluid mechanics / two-phase flow / head-on collision / solitary waves

Sur la collision frontale de deux ondes solitaires pas simulation des équations de Navier-Stokes

Résumé. Ce travail est consacré à l'étude numérique de la collision bidimensionnelle frontale de deux ondes solitaires par simulation numérique des équations de Navier-Stokes en formulation diphasique. L'intérêt de cette étude réside dans la démonstration de la capacité de l'outil numérique original à traiter efficacement ce problème d'onde hautement non-linéaire. Dans cette Note, les résultats numériques sont comparés aux résultats disponibles dans la littérature. Une première investigation de l'hydrodynamique de l'écoulement général est proposée. Il est ainsi démontré que cette configuration est d'une très grande utilité pour valider un code de simulation de mécanique des fluides numérique. (C) 2005 Académie des sciences/Éditions scientifiques et médicales Elsevier SAS

Mécanique des fluides numérique / écoulement diphasique / collision frontale / ondes solitaires

Note présentée par Michel CoMBARnOUS

S1620-7742(01)0????-?/FLA

(C) 2005 Académie des sciences/Éditions scientifiques et médicales Elsevier SAS. Tous droits réservés. 


\section{P. Lubin et al.}

\section{Introduction}

Solitary waves are known for having some interesting properties, such as a symmetrical form with a single hump and a propagation with a uniform velocity without changing form. When simulating two-phase flows, it is important to evaluate the general accuracy of the numerical methods and schemes by checking, for example, the balance of mass and energy in the computing domain. Thus, the results of the different solitary wave analytical theories can be used to compute the initial kinematic properties and simulate the waves propagations in constant depths over horizontal beds in periodic domains [1]. The precision of the simulation is assessed by comparing the free surface shapes and velocities to the theoretical values.

Another major interest is in the type of interaction that can happen between solitary waves, especially the direct, head-on collision between two waves travelling in opposite directions. Maxworthy [2] carried out two kind of wave interaction experiments. After studying the reflexion occurring when a single wave hits a vertical end wall, Maxworthy compared the case of two waves interacting directly. Three main features were outlined from these experiments. First, it has been shown that the waves reach a maximum amplitude greater than the sum of the initial wave amplitudes. Moreover, experimental results showed that a collision has some consequences on the waves (in both wave-wave interactions or end-wall reflexions) such as spatial phase shifts and the shedding of secondary weaker waves following the first. Su and Mirie [3] developed analytical solutions for calculating the effects of the collision, up to the third order of accuracy. They stressed on the fact that the collision does leave imprints on the colliding waves with spatial phase shift and shedding of secondary waves, which is confirmed by Mirie and Su [4]. They also calculated analytically the maximum run-up amplitude reached during the collision of the two solitary waves, as a function of both propagating wave amplitudes.

This head-on collision problem has never been tackled using the Navier-Stokes equations. The interest of this Note is thus twofold. On one hand, we want to prove that simulating head-on collisions between solitary waves can be used as a test-case in order to verify the accuracy of the numerical schemes when solving twophase flow problems. On the other hand, we intend to show the ability of the numerical model to reproduce such a nonlinear problem with a high level of precision concerning the maximum run-up amplitude reached during the collision of the two solitary waves. Furthermore, we want to explain the hydrodynamics of the phenomenon.

However, we will not go any further into the details dealing with the spatial phase shift as both cited authors [2,3] showed discrepancies in the comparison between experimental and analytical results. Moreover, some contradictions are found in the literature. Some authors claim that the solitary waves suffer from permanent loss of amplitude after the interaction $[5,6,7]$. The solitary waves do not recover from a transitional loss of amplitude and thus do not return to their nearly original heights. Some authors tend to prove the contrary $[3,4,8,9]$, the only long-term effect of the collision being the phase shift of the solitary waves. We choose to focus on the preliminary study of the first numerical results, this particular point being of great interest for a perspective work which is now undertaken.

\section{Numerical model}

The incompressible Navier-Stokes equations in their single fluid formulation [10] are used to model the air/water free surface flow at small interface scale in an Eulerian framework. The coupling between velocity and pressure and the divergence free property are solved thanks to an augmented Lagragian method, which has been originaly adapted to two-phase flows by Vincent et al. [11]. In this novel approach, the local penalty term controlling the incompressibility constraint is estimated through a non-dimensional analysis of the physical model. Numerically, a minimization procedure is implemented in order to tackle with the augmented Lagrangian. An Uzawa algorithm is used to solve the associated minimization or penalty problem.

Fixed staggered Cartesian grids ensure an Eulerian representation of free surface flows. The space derivatives of the inertial term are discretized by a hybrid Upwind-Centered scheme and the viscous term is ap- 
proximated by a second order Centered scheme. The implicit time discretization implies the building of a linear system which is solved thanks to an iterative BiCGSTAB II solver, preconditionned under a Modified and Incomplete LU (MILU) technique.

Concerning the single fluid formulation of the motion equations, an advection equation of a phase function $C$ is required to track the free surface evolutions $(C=0$ in the air and $C=1$ in the water). A Volume Of Fluid approach (VOF) is considered to treat the interface tracking. The hyperbolic equation is solved with an explicit Lax-Wendroff TVD scheme (Total Variation Diminishing), which does not require any reconstruction of the phase function.

All the details and references associated to the numerical model can be found in Vincent and Caltagirone $[12,13]$.

The present numerical model has been validated by studying, for example, the simple case of solitary waves propagations in constant depths over horizontal beds in periodic domains [1] or the more complicated cases of three-dimensional plunging breaking waves [14]. This model has been also proved to simulate accurately a solitary wave hitting a vertical end wall [15]. Indeed, Maxworthy [2] considered that, since the system of two head-on colliding waves of equal amplitude is symmetric about the mid-plane, this configuration can be modelled by a single wave hitting a vertical wall.

\section{Head-on collisions between two solitary waves}

Su and Mirie [3] developed analytical solutions for calculating the effects of the collision, up to the third order of accuracy. The subscripts $\mathrm{R}$ and $\mathrm{L}$ refers to the right- and left-going waves heading towards each other, respectively. For two head-on colliding solitary waves with their maximum heights defined as $\epsilon_{R}$ and $\epsilon_{L}$, the maximum run-up $\eta_{m}$ is defined by:

$$
\eta_{m}=\epsilon_{R}+\epsilon_{L}+\frac{\epsilon_{R} \epsilon_{L}}{2}+\frac{3}{8} \epsilon_{R} \epsilon_{L}\left(\epsilon_{R}+\epsilon_{L}\right)
$$

with $\epsilon=H / d, d$ being the water depth and $H$ the waveheight.

\subsection{Initial condition}

We simulate two solitary waves with different amplitudes propagating towards each other in a $20 \mathrm{~m}$ long and $0.6 \mathrm{~m}$ high periodic domain. This configuration increases the level of difficulty as we will see the waves colliding several times during the simulation, the waves moving out of the domain on one side to re-enter on the other side. This complexity will thus allow us to put in evidence the high level of accuracy of our numerical method. The numerical domain is discretized into $1000 \times 150$ regular Cartesian cells $\left(\Delta x=2 \cdot 10^{-2} m\right.$ and $\Delta z=4 \cdot 10^{-3} \mathrm{~m}$ ). A symmetry boundary condition in the lower limit and a free boundary condition in the upper limit are imposed. The simulation time step is chosen to verify the stability criterion (Courant-Friedrichs-Levy) less than one for the interface algorithm. We run 3250 iterations, which corresponds to $27 \mathrm{~s}$ of propagation. All calculations are made using the values of the densities and the viscosities of air and water, as: $\rho_{a}=1.1768 \mathrm{~kg} \cdot \mathrm{m}^{-3}$ and $\rho_{w}=1000 \mathrm{~kg} \cdot \mathrm{m}^{-3}, \mu_{a}=1.85 \times 10^{-5} \mathrm{~kg} \cdot \mathrm{m}^{-1} . \mathrm{s}^{-1}$ and $\mu_{w}=1 \cdot 10^{-3} \mathrm{~kg} \cdot \mathrm{m}^{-1} . \mathrm{s}^{-1}$.

Figure 1 should be here

The wave crests are initialized far apart from each other at $x_{R}=5 \mathrm{~m}$ and $x_{L}=15 \mathrm{~m}$ (Fig. 1). The water depth is $d=0.302 \mathrm{~m}$, the waveheights are $\epsilon_{R}=0.11$ and $\epsilon_{L}=0.33$, which give the dimensional amplitudes $H_{R}=0.03322 \mathrm{~m}$ and $H_{L}=0.1 \mathrm{~m}$, so the crests ordinates are located at $z_{R}=0.33522 \mathrm{~m}$ and $z_{L}=0.402 \mathrm{~m}$, respectively. The initial wave celerities are $c_{R}=1.8134 \mathrm{m.s} \mathrm{s}^{-1}$ and $c_{L}=1.9859 \mathrm{m.s} \mathrm{s}^{-1}$, as predicted by the first order theory [16, 1]. From Eq. (1), the maximum theoretical run-up is $\eta_{\mathrm{m}}=0.1349 \mathrm{~m}$, which gives a maximum ordinate $z_{m}=0.4369 \mathrm{~m}$. The waves are assumed to meet for the first time at $t=2.7 \mathrm{~s}$ and $x_{1 \text { st }}$ collision $=9.8 \mathrm{~m}$. During the 3250 iterations ( $27 \mathrm{~s}$ of propagation), the waves will propagate and collide each other four times. 


\section{P. Lubin et al.}

\subsection{Flow description}

We predicted that the abscissa of the first collision is $x_{1^{\text {st }} \text { collision }}=9.778 \mathrm{~m}$, which agrees with the theoretical value $x=9.8 \mathrm{~m}$, as shown in Fig. (2c). We illustrate in Figs. (2) the first collision in details. Both waves propagate towards each other and seem to merge into a unique wave, which amplitude is higher than the sum of both incoming waves (Fig. 2c). The profile looks like a "single wave". During the interaction, we could observe that large vertical accelerations are developed until the merging wave reached its maximum amplitude. At the instant when the vertical velocity vanishes, the kinetic energy is nil and the potential energy reaches its maximum magnitude, the unique wave seems to be at rest. Then it starts to fall down, both waves splitting apart to recover their original characteristics. But it can be observed in Fig. (2f) that the left-going wave seems to be slightly distorted, which is consistent with the discussions of Maxworthy [2] and Su and Mirie [3]. The left-going wave looks unsymmetrical and appears to propagate with a slightly higher celerity than its initial one. As pointed out by Maxworthy [2], the left-going wave is steeper than originally and propagates faster until it recovers its original shape. Then it reorganizes itself before colliding with the right-going wave another time. We can clearly see a weak wave following the left-going wave, propagating in the same direction. These observations are not so easy to do with the rightgoing wave because of its low amplitude. The waves collide four times, due to the periodic configuration of the numerical domain. The resulting flow gives a general impression of spasmodic movement because of the left-going wave which is subjected to accelerations and decelerations between each collision and the slow motion of the two waves coming together.

Figure 2 should be here

During the 3250 iterations, the right-going wave should propagate along $48.96 \mathrm{~m}$ and the left-going wave should propagate along $53.62 \mathrm{~m}$. These theoretical distances correspond to the abscissae the waves should have reached in the case they would not have been interacting. We checked that the waves passed more than two times by their initial position propagating without major distortion. However, we recorded that the right-going wave propagated along $48.3 \mathrm{~m}$ and the left-going wave along $52.2 \mathrm{~m}$. This corresponds to a translation of $0.66 \mathrm{~m}$ and $1.42 \mathrm{~m}$, as compared to the respective values for the distance of propagation, simply estimated with the initial celerities and the time of simulation. This difference is due to the time delay during their interaction and, also, possibly due to the numerical dissipation of our model.

The right-going wave loses $1.10^{-3} \mathrm{~m}$ of its amplitude after each collision, the amplitude at the end being $z=0.334 \mathrm{~m}$ (so a total loss of $1.22 \times 10^{-3} \mathrm{~m}$ or $3.6 \times 10^{-1} \%$ ). The left-going wave loses $8.10^{-3} \mathrm{~m}$ after the first collision, then loses $3.10^{-3} \mathrm{~m}$ on average, the amplitude at the end being $z=0.384 \mathrm{~m}$ (so a total loss of $1.6 \times 10^{-2} \mathrm{~m}$ or $4 \%$ ). These successive losses in amplitudes put in evidence the fact that each collision gives birth to secondary dispersive waves travelling in the same direction of the wave which they come from. The shedding of secondary waves is enhanced at higher amplitudes. This point has been discussed by Maxworthy [2]: the higher the wave is, the higher the secondary shed wave will be. This is confirmed by the fact that the right-going wave loses less amplitude than the left-going one. The numerical error has been evaluated for the mass conservation and found to be less than $4.8 \times 10^{-2} \%$, the initial volume being $v o l_{i n i}=6.271 \mathrm{~m}^{2}$. Table (1) is a summary of each calculated maximum amplitudes, considering the loss of amplitude for each wave after each collision.

Table 1 should be here

\section{Concluding remarks and perspectives}

The numerical model, which has been presented, is thus able to take accurately into account highly complex nonlinear phenomena such as solitary waves interactions. The precision of the simulation is assessed by comparing the numerical results with experimental and analytical results, which makes this configuration interesting to be considered as a test case. The present results agree with the observations made by Maxworthy [2] and Su and Mirie [3]. It has been observed that the collisions induce imprints to the waves, the waves propagating faster after each collision and losing amplitude. As we ensure ourselves about the 
On the Navier-Stokes equations simulation of the head-collision between two surface solitary waves

capability of our numerical methods to handle with the head-on collision problem, we are now having a close inspection on the phase shift phenomenon, as the refereed authors did not agree in their results.

A systematical study of the configuration with two solitary waves of same amplitudes colliding is also considered, as Maxworthy [2] left the discussion open about the opportunity to do the analogy between two solitary waves of same amplitudes interacting directly and the wave-reflexion case.

Our main interest is to use our numerical model to work on appropriate head-on collisions configurations to investigate whether or not the solitary waves suffer from permanent loss of amplitude after the interaction, in order to discuss with the contradictory results found in the literature.

Acknowledgements. The authors would like to acknowledge the financial and scientific support of the french INSU - CNRS (Institut National des Sciences de l'Univers - Centre National de la Recherche Scientifique) program "Programme Atmosphère-Océan Multi-échelle" (PATOM) and the IDRIS-CNRS (Institut du Développement et des Ressources en Informatique Scientifique) and CINES (Centre Informatique National de l'Enseignement Supérieur) for their material support (project ter 2237). We also thank Pr. Mohamed Rady, for his critical reading that guided us in improving this article, and Pr. André Temperville, for the valuable suggestions and references.

\section{References}

[1] P. Lubin, H. Lemonnier, Propagation of solitary waves in constant depths over horizontal beds, Multiphase Science and Technology, 16 (2004), 237-248.

[2] T. Maxworthy, Experiments on collisions between solitary waves, J. Fluid Mech., 76 (1976), 177-185.

[3] C. H. Su, R. M. Mirie, On head-on collision between two solitary waves, J. Fluid Mech., 98 (1980), 509-525.

[4] R. M. Mirie, C. H. Su, Collisions between two solitary waves. Part 2. A numerical study, J. Fluid Mech., 115 (1982), 475-492.

[5] D. P. Renouard, F. J. Seabra-Santos, A. M. Temperville, Experimental study of the generation, damping, and reflexion of a solitary wave, Dynamics of Atmospheres and Oceans, 9 (1985), 341-358.

[6] J. G. B. Byatt-Smith, On the change of amplitude of interacting solitary waves, J. Fluid Mech., 182 (1987), 485-497.

[7] J. G. B. Byatt-Smith, The reflection of a solitary wave by a vertical wall, J. Fluid Mech., 197 (1988), 503-521.

[8] J. D. Fenton, M. M. Rienecker, A Fourier method for solving nonlinear water-wave problems: application to solitary-waves interactions, J. Fluid Mech., 118 (1982), 411-443.

[9] J. L. Bona, M. Chen, A Boussinesq system for two-way propagation of nonlinear dispersive waves, Physica D, 116 (1998), 191-224.

[10] R. Scardovelli, S. Zaleski, Direct numerical simulation of free-surface and interfacial flow, Annu. Rev. Fluid Mech., 31 (1999), 567-603.

[11] S. Vincent, J.-P. Caltagirone, P. Lubin and T. N. Randrianarivelo, An adaptative augmented Lagrangian method for three-dimensional multi-material flows, Computers and Fluids, 33 (2004), 1273-1289.

[12] S. Vincent, J.-P. Caltagirone, Efficient solving method for unsteady incompressible interfacial flow problems, Int. J. Numer. Meth. Fluids, 30 (1999), 795-811.

[13] S. Vincent, J.-P. Caltagirone, A One Cell Local Multigrid method for solving unsteady incompressible multi-phase flows, J. Comput. Phys., 163 (2000), 172-215.

[14] P. Lubin, S. Vincent, J.-P. Caltagirone, S. Abadie, Fully three-dimensional direct simulation of a plunging breaker, C. R. Mécanique, 331 (2003), 495-501.

[15] P. Lubin, Large Eddy Simulation of plunging breaking waves, $\mathrm{PhD}$ thesis (in english), Université Bordeaux 1 (2004).

[16] J.-J. Lee, J. E. Skjelbreia, F. Raichlen, Measurements of velocities in solitary waves, J. of Waterway, Port, Coastal, and Ocean Eng., WW2 108 (1982), 200-218. 


\section{P. Lubin et al.}

\begin{tabular}{|c|c|c|c|c|}
\hline Collisions & $z_{m}(m)$ (theory) & $z_{m}(m)$ (numerical results) & Error $(\mathrm{m})$ & Error $\%$ \\
\hline $1^{\text {rst }}$ & 0.4369 & 0.4372 & $3.10^{-4}$ & $7.10^{-2} \%$ \\
\hline $2^{\text {nd }}$ & 0.4279 & 0.4301 & $2.10^{-3}$ & $5.10^{-1} \%$ \\
\hline $3^{\text {rd }}$ & 0.4229 & 0.4247 & $2.10^{-3}$ & $4.10^{-1} \%$ \\
\hline $4^{\text {th }}$ & 0.4215 & 0.4233 & $2.10^{-3}$ & $4.10^{-1} \%$ \\
\hline
\end{tabular}

TAB. 1 - Maximum run-up ordinates reached for each collision.

List of tables 
On the Navier-Stokes equations simulation of the head-collision between two surface solitary waves

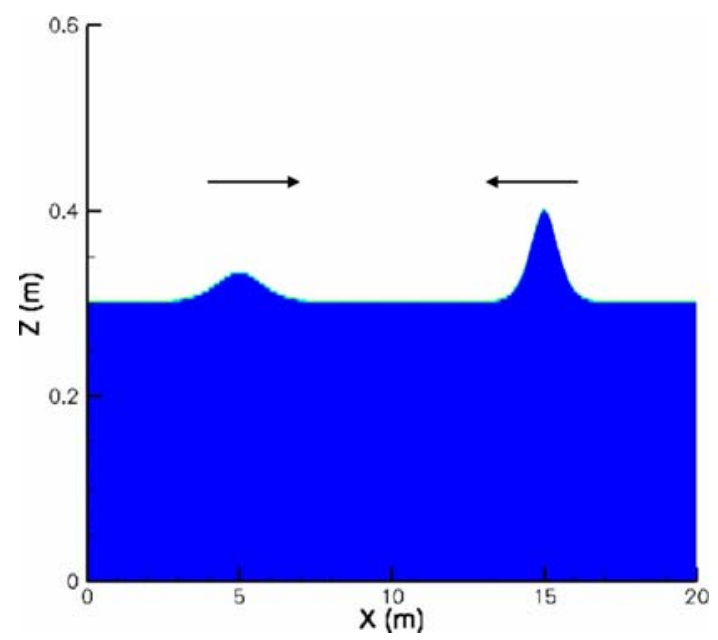

FIG. 1 - Initial condition for the head-on collisions test-case. $t=0 s, \epsilon_{R}=0.11$ and $\epsilon_{L}=0.33, C>0.5$. List of figures 
P. Lubin et al.

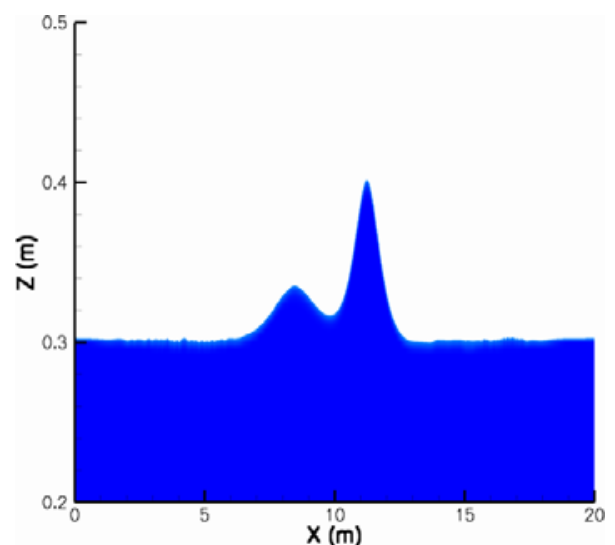

a. $\mathrm{t}=1.9 \mathrm{~s}$

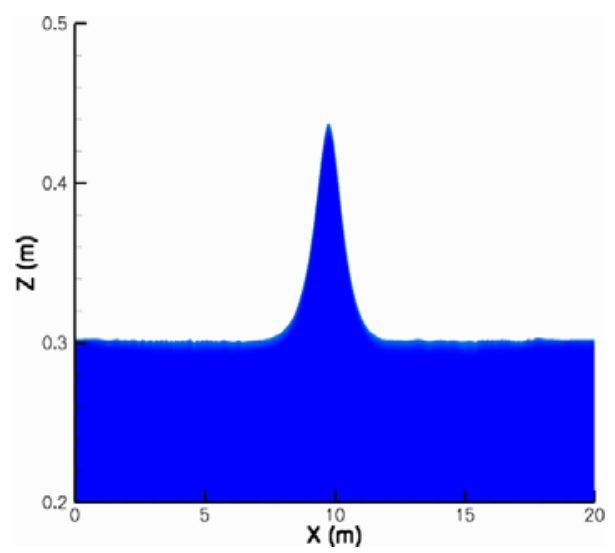

c. $t=2.7 \mathrm{~s}$

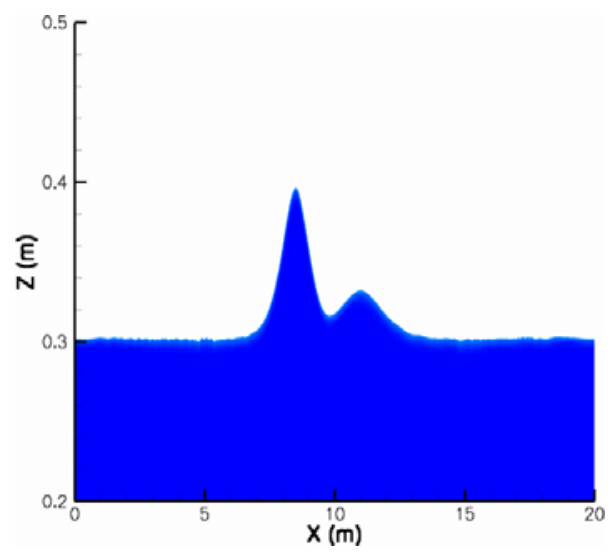

e. $t=3.4 \mathrm{~s}$

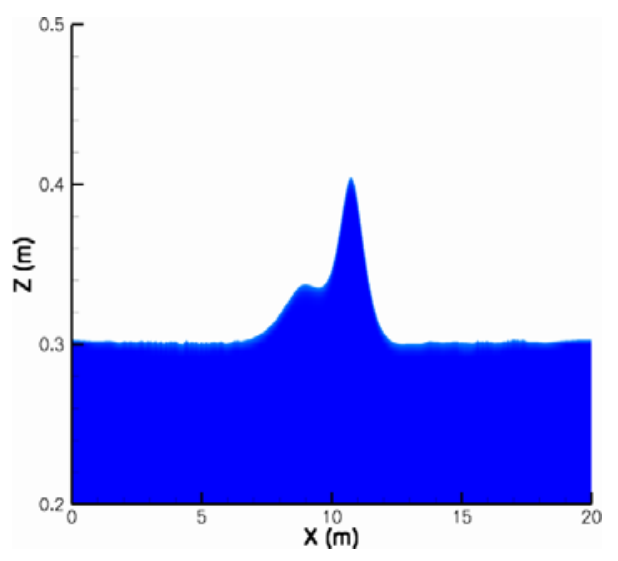

b. $t=2.2 \mathrm{~s}$

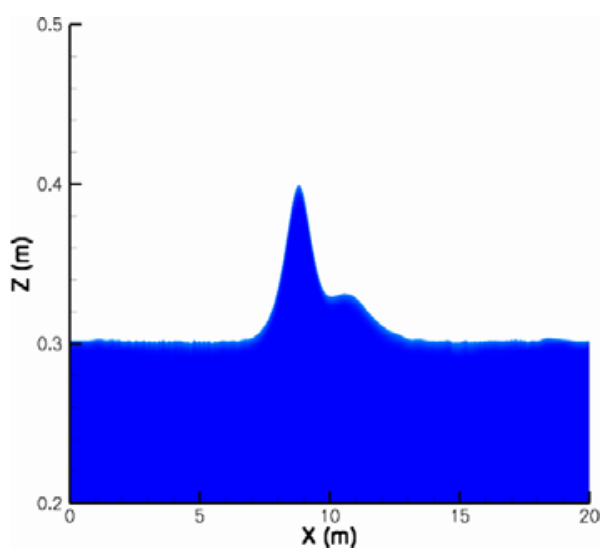

d. $\mathrm{t}=3.2 \mathrm{~s}$

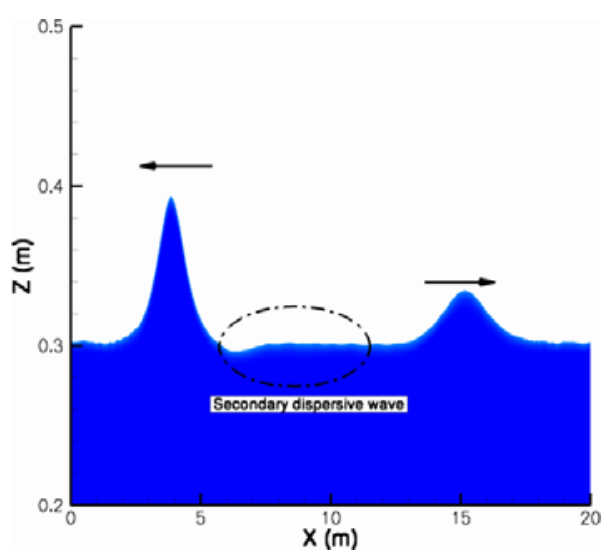

f. $t=5.7 \mathrm{~s}$

FIG. 2 - Details of the first collision at various instants. $C>0.5$. 